This item was submitted to Loughborough's Research Repository by the author.

Items in Figshare are protected by copyright, with all rights reserved, unless otherwise indicated.

\title{
Manufacturing system engineering moderator: an aid for multidiscipline project teams
}

PLEASE CITE THE PUBLISHED VERSION

http://dx.doi.org/10.1080/0020754031000077257

PUBLISHER

(c) Taylor \& Francis

VERSION

AM (Accepted Manuscript)

LICENCE

CC BY-NC-ND 4.0

\section{REPOSITORY RECORD}

Harding, Jennifer A., K. Popplewell, and D. Cook. 2019. "Manufacturing System Engineering Moderator: An Aid for Multidiscipline Project Teams”. figshare. https://hdl.handle.net/2134/9805. 
This item was submitted to Loughborough's Institutional Repository (https://dspace.lboro.ac.uk/) by the author and is made available under the following Creative Commons Licence conditions.

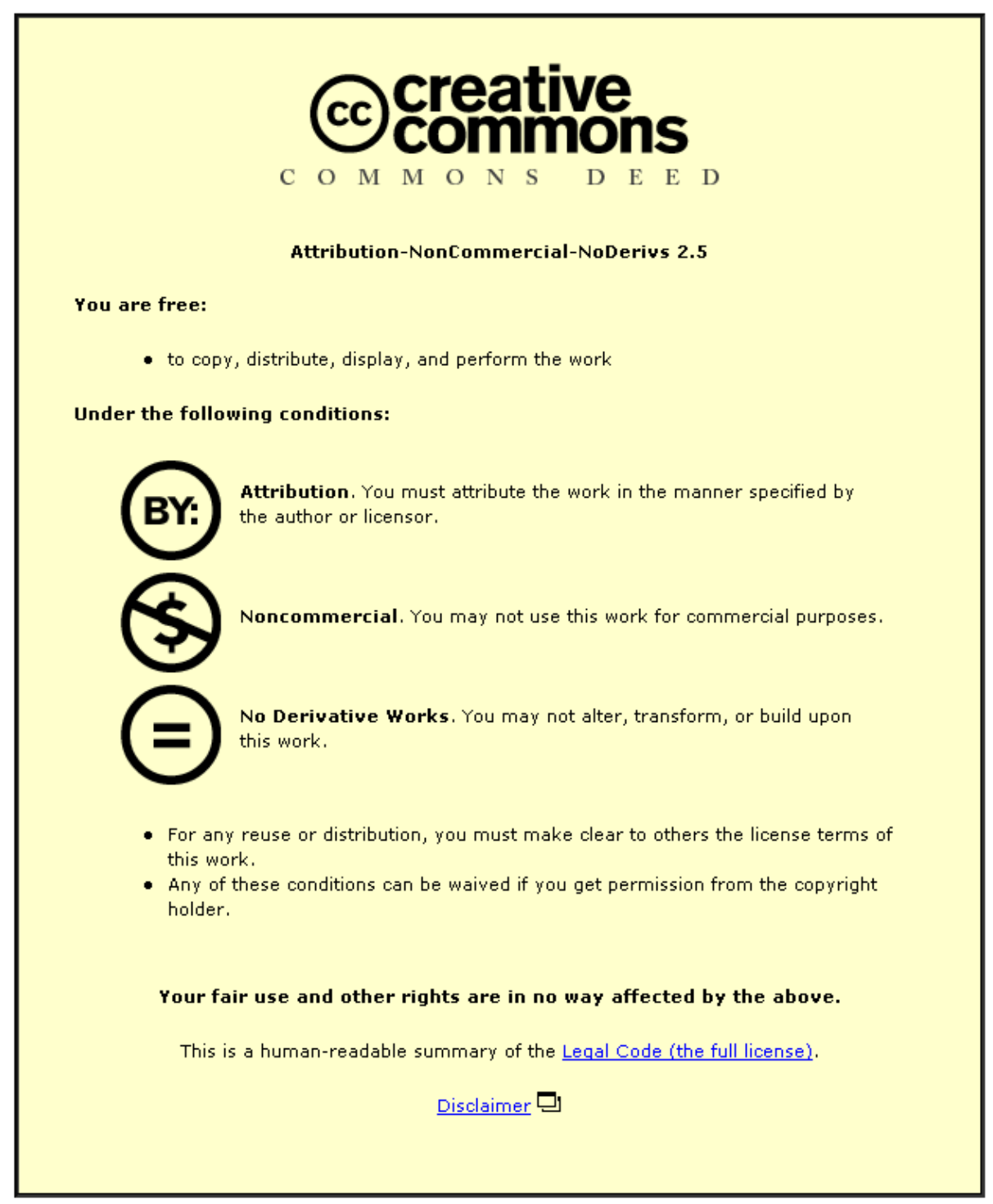

For the full text of this licence, please go to: http://creativecommons.org/licenses/by-nc-nd/2.5/ 


\title{
A Manufacturing System Engineering Moderator: an aid for multi-discipline project teams
}

By

\author{
J A Harding \\ Wolfson School of Mechanical and Manufacturing Engineering, \\ Loughborough University \\ K Popplewell \\ School of Engineering, \\ Coventry University \\ D Cook \\ Kirby Computing Ltd
}

\begin{abstract}
Manufacturing System Engineering (MSE) is a complex process generally performed by a multi-discipline project team. The Manufacturing System (MS) must satisfy many different requirements and objectives so compromises generally have to be made to achieve a balanced design for the new or re-engineered MS. Project team members must be aware when decisions are made which are significant to other team members. When teams are large and located in multiple sites, this can be very difficult to achieve, and intelligent support systems are necessary. The MSE Moderator is designed to monitor design decisions, evaluate their significance to individual project team members and communicate with any team members deemed necessary.
\end{abstract}

Keywords: Manufacturing System Engineering, Manufacturing System Design, Intelligent support systems, Multi-discipline teams, Distributed Teams

\section{Introduction}

A manufacturing system may be engineered, or re-engineered for a variety of different reasons. Projects may range from partial or comprehensive overhauls of existing facilities, to complete designs of new manufacturing operations. The competitive market forces manufacturing enterprises to accelerate new product design and launch, as reduced time to market may be critical to the eventual success of the product. To facilitate the smooth transition from design to manufacture, it is essential that the manufacturing system which will eventually produce the product is considered in parallel with the product design. This may lead to the manufacturing system being fully or partially designed in parallel with the product design. In contrast, another common example of manufacturing system re-engineering occurs when the manufacturing system consists of a supply chain. Supply chains inevitably require modification, or re-engineering if alternative companies or facilities within a parent company can offer improved benefits to the chain, or if for some reason, existing links 
cease to work effectively within the chain. Similarly, a globally distributed operation may need to engineer a completely new manufacturing system when they plan to open more cost-effective facilities in different parts of the world, since this may necessitate designing a complete manufacturing plant.

Methods for manufacturing system design are not well understood or documented, although substantial amounts of work exist in the parallel field of enterprise and manufacturing system modelling (Harding et al, 1999, Yu et al, 2000, ESPRIT, 1993, Scheer, 1992, Bouti and Ait Kadi, 1998). Manufacturing systems are complex, and projects often require expert contributions from many different disciplines to successfully design or modify the system. The Manufacturing System Engineering (MSE) process has been examined as part of the MISSION Research Project (IMS/ESPRIT 29656) (Rabe, 2000, Mission Consortium, 2001) which has recently been completed as part of the Intelligent Manufacturing Systems (IMS) program. In particular, the MISSION research examined many applications of simulation within manufacturing system design and operation. A key area of the project also examined the provision of intelligent support applications within the MSE process. This paper reports on the research results for one particular intelligent support application, the MSE Moderator. The MSE Moderator monitors the progress of, and inputs to the MSE process and ensures that project members are made aware of additions or changes to the manufacturing system design which are relevant to their rôle in the MSE process. This is a complex and important function, particularly as MSE projects commonly utilise multi-discipline, distributed teams.

\section{MSE Moderator Concept \\ 2.1 Multi-Discipline Design}

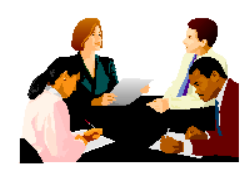

Small Co-located Team

High Level of Aw areness

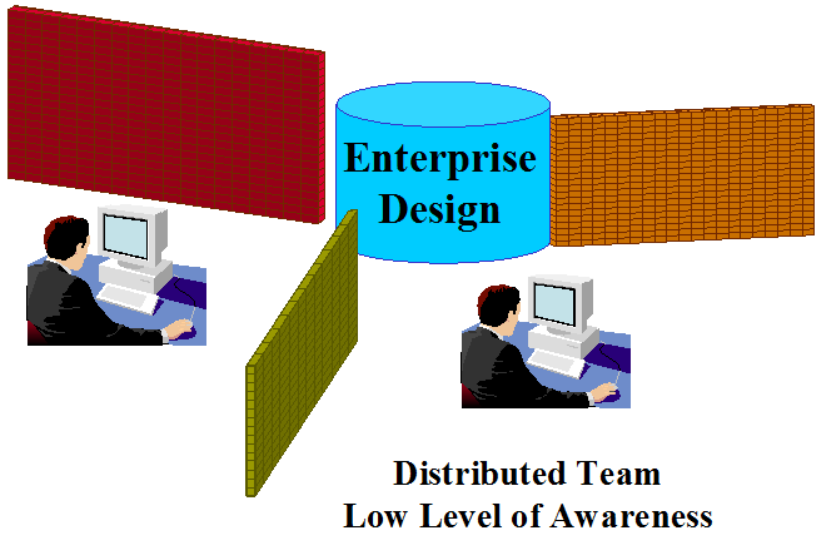

Figure 1: Distributed Teams reduce awareness and informal information exchange 
A manufacturing system must satisfy many different requirements and objectives. To achieve this, it is usual for a project team to be formed, which utilises the skills of many different team members from a variety of disciplines, each with a particular expertise and background experiences. Individual team members may, for example, be skilled in areas such as process selection, equipment selection, facility layout, performance prediction (perhaps by simulation), and potentially many others. In most design activities, compromises have to be made to achieve a balanced design which meets all the requirements and objectives of the project. However, if individual experts work on their own on particular aspects of a design, it may be difficult to identify when compromises need to be made (see figure 1). These difficulties are intensified as large manufacturing organisations commonly use project teams with members located at different sites (possibly even in different countries).

\subsection{Functions of an MSE Moderator}

The primary function of the MSE Moderator is to raise awareness within and between members of the project team. It makes project team members aware when they take a design decision which is significant to another team member (or members) and project team members are also made immediately aware when a decision has been made which affects them. It is much easier for project managers to maintain an environment of common understanding when project team members can meet together regularly, exchange information and discuss progress. When project teams are large and distributed, possibly only meeting through exchanges of emails or telephone conferences, additional information technology and intelligent support systems, such as the MSE Moderator, are necessary.

The various activities performed by the MSE Moderator to achieve its primary function are termed the Moderation Process, and the essential constituent activities are listed below.

1. The MSE Moderator must know whenever a decision is taken relating to the MSE project, as this is equivalent to a change being made to the MS design.

2. The MSE Moderator must be able to evaluate the change and determine whether the decision is significant to any other team members

3. The MSE Moderator must be able to communicate with any project team member who is assessed as being affected significantly by the change.

Additional necessary functionality for the MSE Moderator can be identified through examination of the activities in the Moderation Process. For example, the MSE Moderator must be able to collect, store and evaluate knowledge about what is important (significant) to individual design team members. Also, it must be straightforward to modify this knowledge during the course of a project as team members may join or leave the project and the relevance of particular types of decision may also change. The MSE Moderator must also be able to store, or have access to, contact information for project team members.

The activities described above have been termed the Knowledge Acquisition Process.

Clearly any implementation of the MSE Moderator has substantial information and knowledge requirements to enable the moderation process to take place successfully, but many different approaches to the implementation are possible, and these are partly 
dependent upon the environment in which the MSE Moderator is to work. For example, the performance of activity 1 partly depends on how decisions are recorded and how design information is stored. If design information is recorded in relational or other conventional databases, then the MSE Moderator could regularly interrogate the database. Alternatively, in an object oriented system, the MSE Moderator could be activated by messages being passed when design information has been changed, the mechanism for transmitting such messages being embedded in the highest inheritance level of methods updating database content. The methodology used in the current research has built on previous experiences gained in the area of product design (Harding \& Popplewell, 1996). The remainder of this paper will report on the design and implementation of the prototype MSE Moderator demonstrated at the completion of the MISSION Research project. The concept of the Knowledge Representation Model (KRM) is presented first, as this provides a basis for the structure of the prototype MSE Moderator. The KRM was first introduced by Harding (1996) and has subsequently provided a basis for several intelligent systems to support various aspects of design (Omar et al, 1999, Borja et al, 2001).

\section{Specification}

\subsection{Storing Knowledge and Expertise}

Substantial amounts of information and knowledge about the MSE project objectives, the expertise and skills of the project team members, and the manufacturing system being engineered, must be available for the MSE Moderator to successfully perform its moderation processes. Many different types of expertise must be supported, in a variety of different situations that may arise during the design process (Harding \& Popplewell, 1996). There is no single method which can best capture or store the information and knowledge which must be utilised to enable such experts to work effectively, since the content of this knowledge is so varied. It is therefore essential that information and knowledge are captured in as flexible a manner as possible, to ensure its reusability in a variety of situations, by a variety of users or applications over a period of time.

The potential to reuse knowledge from previous projects is also a very important consideration. Information and knowledge (often gained through experience on past projects) are very valuable resources. It is no longer acceptable for such valuable resources to exist only in the experience of long established employees, since industry cannot afford to lose its specialist knowledge as employees retire or leave for other reasons. Information models, which can be instanced as populated databases providing a single representation of product related data, are central to many research activities intended to support product design and development (Krause et al 1993, McKay et al 1996). Valuable information about manufacturing capabilities and facilities can also be modelled and used for many design and operation activities (Molina and Bell, 1999, Yu et al, 2000). However, these generally only satisfy part of industry's requirements, since ways to capture the knowledge of how to utilise and apply such information are also of vital importance. If this can be achieved industry can preserve much of its valuable design experience over successive generations of employees.

Many artificial intelligence paradigms and existing systems were considered for the implementations of the moderator, and they all provide particular benefits in certain 
conditions. However, no existing system was found which easily satisfied the following over riding requirements of the Moderator:

- It must be able to easily access information from existing databases (of various types).

- It must be able to communicate easily with project team members.

- It must be quick and easy to maintain and change knowledge which is being used in the moderation process.

- It must be quick and easy to reuse knowledge.

A combination of object technology, database technology and established programming languages (such as $\mathrm{C}++$ ) can easily satisfy these individual requirements. $\mathrm{C}++$ code can also be used to activate neural networks, genetic algorithms or other intelligent systems as required, to gain the benefits of these approaches when necessary. The KRM modelling platform was built on these technologies to specifically address the above requirements.

\subsection{The Knowledge Representation Model (KRM) Concept}

The KRM is a flexible hybrid approach, which enables a variety of artificial intelligence representations of knowledge to be used, and reused. The KRM provides a framework, and class hierarchy, and using this approach knowledge can be captured used, and maintained as persistent objects that are easily accessible in a database.

Using the KRM concept, knowledge is represented as groups of associated, interactive objects, which are termed as modules. The knowledge is processed as the objects pass messages to each other. The KRM objects can exhibit many different types of behaviour when they receive a message, and their activities can include: collecting information; processing information; checking the truth of specified conditions; communicating with users; activating neural networks; etc. As this range of possible activities is virtually limitless, the potential applications of the KRM are numerous and varied. The KRM can be used to produce knowledge bases, by storing the objects as persistent objects within an object oriented database. It is however essential that a true object oriented database, which supports processing of object methods (or member functions), inheritance and polymorphism be used. As the KRM concept makes use of database technology, and KRM objects are able to collect and process information, the KRM is further enhanced when used in conjunction with information models, as this facilitates the processing of product or manufacturing information.

The KRM concept enables knowledge to be represented by one or more modules hence, each type of expertise considered by the MSE Moderator can be stored as a module, in an object oriented database. In the case of the MSE Moderator, knowledge about individual project members, and the knowledge about what changes are important to them, and what actions should be taken if such changes occur, are stored in objects called Design Agent Modules. Each Design Agent Module knows how to process its own knowledge, as this behaviour is implemented in methods of the class. The inter-active objects that are associated with each Design Agent Module come 
from various classes, including Ruleset, Rule, Condition and Action objects, and the processing of knowledge is achieved by message passing between instances of these various classes. The names used to identify classes associated with the MSE Moderator and Design Agent Modules are very similar to the terminology of standard expert systems, in particular of production systems. This is a deliberate use of metaphor, as it is believed that terms such as Ruleset, Rule, WorkingMemory, etc are sufficiently well known for some level of understanding of the primary functions of these objects to be immediately gained by any casual reader. However, it is important that the metaphor is not taken too far and the approach over-simplified as this can lead to misunderstanding of the KRM. The KRM is not a production system. The KRM is an object oriented system, and the use of object technology in software systems based on the KRM is fundamental to the ultimate success of the implemented systems. Implementations of the KRM work with classes of objects, so instances of these classes have state, behaviour and identity, and therefore the objects provide all the flexibility and power and potential for reuse of object oriented systems. By making extensive use of inheritance structures and polymorphism, it has been possible for the similarity of behaviour of certain classes of objects to be exploited even though the implementations of particular sub-classes of objects is significantly different. This point is central to the concepts of this work.

\subsection{MSE Moderator as an Instance of the KRM}

The MSE Moderator is an intelligent, knowledge based system which supports the collaboration and co-ordination of many different types of expertise through a variety of different situations which arise during the MSE process.

Many different types of knowledge and expertise may contribute to the design of a manufacturing system and the MSE Moderator must be able to store knowledge of what aspects of the design are important to any or all of these participants. In addition, team members who can contribute constructively to the manufacturing system design process, inevitably vary throughout the lifecycle of the project, as different skills are required at different stages of the project. The knowledge content of the MSE Moderator must therefore be directly determined by the range of experts working in the project team at any particular time. It is not possible to develop a hard-coded representation of the knowledge needed for a general implementation, for at least two reasons:

1.The knowledge used by the MSE Moderator will be highly dependent on the organisational structures and practices used both within a particular project, and used generally within the enterprise or enterprises that are undertaking the manufacturing system design. The knowledge base will therefore differ between one implementation and another, and indeed it is likely that only the highest level of the structure of the MSE Moderator will be constant between implementations.

2. It is highly probable that the knowledge base will need to change as a project progresses, since experts will join or leave the project, requiring changes in the knowledge that is available to the MSE Moderator.

These points are emphasised here as they are very important when deciding on how knowledge about the project team members and design issues that are relevant to them should be stored. It is essential that the MSE Moderator can capture and store 
information and knowledge about the project members, in a very flexible, versatile manner, that ensures its reusability in a variety of situations, by a variety of users or applications over a period of time.

The MSE Moderator has been implemented with a separate knowledge base, based on the KRM, which was originally designed to support distributed team working, as exemplified by design teams in concurrent engineering projects (Harding, 1996). Instances of the KRM may be used to capture different types of expertise within an object oriented database. This approach enables knowledge to be stored in database structures alongside product and manufacturing information, thus enabling both information and knowledge to be shared, and reused, by different applications.

\section{Prototype Implementation of the MSE Moderator 4.1 MISSION Research Project}

The MISSION project research (IMS/ESPRIT 29656) was directed at establishing new ways of using simulation for MSE, and to understanding the mechanisms and concepts necessary for successful application of information modelling and simulation techniques for global MSE activities. The general goal of MISSION was the support of the MSE process by integrating tools that can be employed in various aspects of the MSE process. As described in the MISSION Project final report, D24 (Mission Consortium, 2001), the MISSION concept has been embodied as the Mission Modelling Platform (MMP). This includes two distinct, but linked environments, the Manufacturing System Run Time Infrastructure (MS-RTI), which supports execution of distributed simulations, and the MSE Integration Infrastructure, which provides the mechanism for interfacing $\boldsymbol{M S E}$ Agents that participate in the MSE Process. The purpose of the MSE integration environment is to allow, and support information sharing amongst any or all MSE Agents that may have an interest in that information. Any information relating to the manufacturing system or to the MSE project can be structured according to the MMP information model and stored in databases. Access to the databases is provided and supported by an MSE Agent called the Information Manager.

In the MISSION project, the term "MSE Agent" is used to refer to each combination of engineer(s) and supporting software performing an identifiable function to contribute to the developing MSE design. The developing design is shared between MSE Agents, and is held in a common MSE database, structured by a MISSION information model to meet the project's needs. Thus, for example, the project management function may be fulfilled by a Project Agent which may be expected to include software tools to support both strategic management and project planning, whilst simulation software and statistics packages would be available within a Simulation Agent, to provide performance evaluation. It is important to note that using this definition, the balance of human and computer intelligence in an MSE agent will vary between MSE agents. Few MSE agents will be entirely software based, and no MSE agent can be entirely human as there must be at least an interface with the MSE database.

The MISSION platform is particularly valuable in supporting simulation applications resulting from the development of globally distributed or virtual enterprises (Rabe 2000). 


\subsection{Mission Architecture}

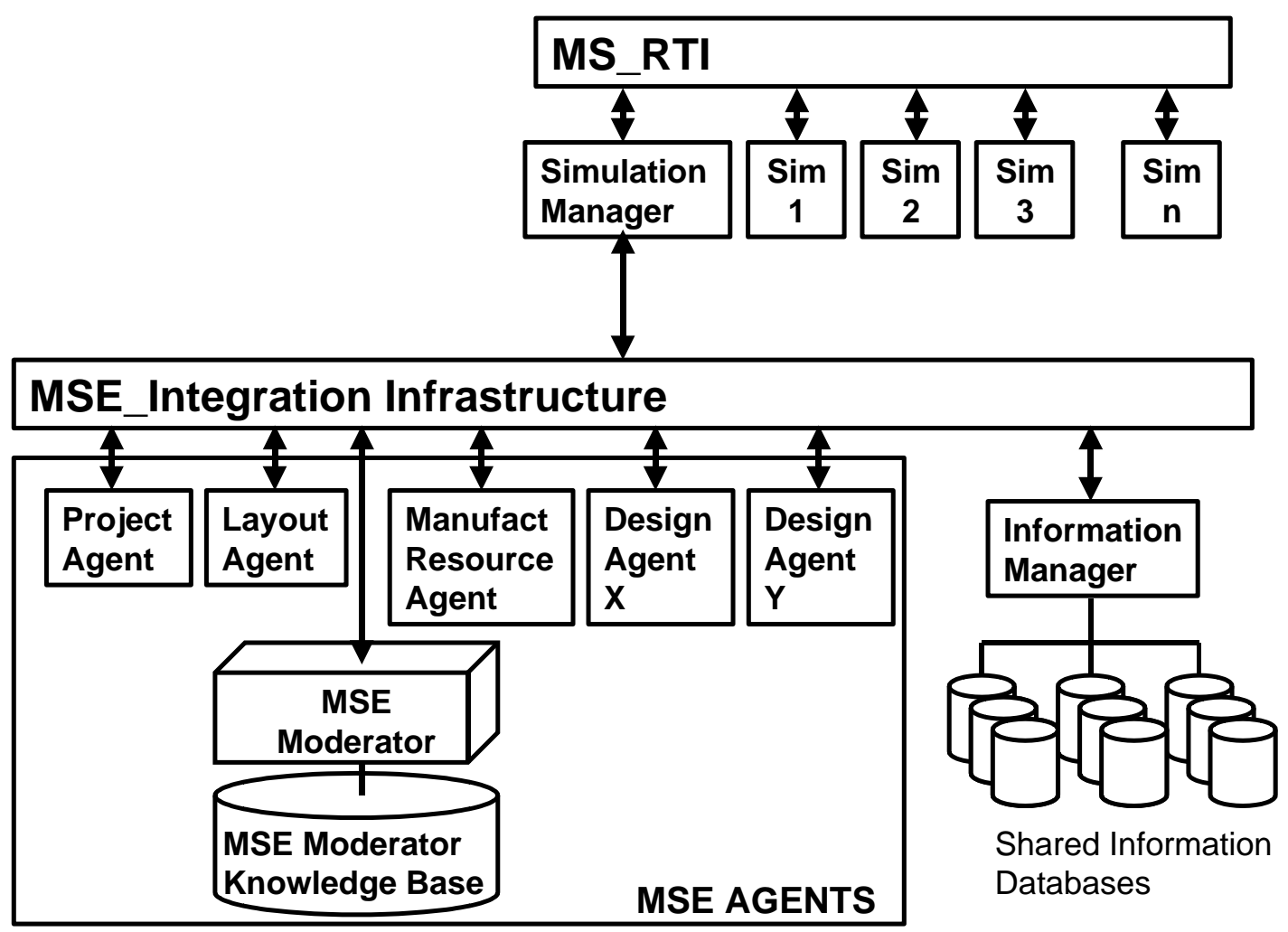

Figure 2: Mission Modelling Platform (MMP)

The MISSION Modelling Platform (MMP), provides a two level infrastructure to support both specialist manufacturing system design tools and distributed simulators to support system evaluation and performance measurement. This is shown in the outline of the MMP that is provided in figure 2.

The Manufacturing System Run Time Infrastructure (MS-RTI) supports execution of distributed simulations. The components of the distributed interface are specified by the Simulation Manager, who also determines which simulation runs are necessary, and accepts output from the runs. In the future, it is envisaged that the MS-RTI will provide access to real production systems, such as process planning and control systems and shop floor control systems, as these operational systems develop and incorporate their activities into the simulation. Within this platform, time synchronisation mechanisms (independent from calendar time) are essential for individual simulators running in parallel to interact. The MSE Integration Infrastructure provides the mechanism for interfacing MSE Agents. The intention is that the MSE integration infrastructure is open so that the set of MSE agents working in this environment, can be increased or changed, depending on the requirements of the project. Other agents could be attached to extend the range of manufacturing systems engineering activities integrated, so the set shown in figure 2 is simply an example, and could never be definitive or complete. 
In the prototype MMP, information relating to the manufacturing system being designed was stored in Oracle databases and access and sharing was orchestrated by the Information Manager. However, since the MSE Infrastructure is open, an Information Manager could be written to support any implementation of an MSE design data repository, provided only that it meets the interfacing requirements of the Infrastructure.

\subsection{Implementation of MSE Moderator within MISSION Platform}

The implementation of the MSE Moderator will now be illustrated using the following example Manufacturing System Design Scenario:

A large, globally distributed manufacturing enterprise is using the MMP to design a new manufacturing system for the manufacture of a new product. The Project Agent has been used to specify details of the project team members, to identify the products to be produced by the new system, and to record the initial objectives for the system. These include the average number of products to be made per week, the maximum number of products required in any week, the maximum variable cost for the product, the delivery policy (eg from stock, or within 10 days of order, or...), etc. The Project Agent has communicated with the Information Manager, through the MSE Integration Infrastructure, and has stored this information in the shared information databases (see figure 2). The current project team also includes a Manufacturing Resource Agent, who has expertise in the specification and allocation of production machinery and other essential resources and facilities for the particular processes involved in the manufacture of this and similar product varieties. A Layout Agent, who has expertise in the specification and design of the layout for production lines and all support areas, is also included in the team.

\subsubsection{Knowledge of MSE Agents}

As previously explained, the MSE Moderator designed during the MISSION research, is based on the KRM concept. The knowledge base for the MSE Moderator is implemented and stored as an ObjectStore database, containing persistent objects from several different classes. Primarily, knowledge of MSE Agents is structured using the class Design Agent Module. Knowledge about each individual MSE Agent and the knowledge about what changes are important to it, and what actions should be taken if such changes occur, is stored in a separate Design Agent Module object.

Each Design Agent Module object knows how to process its own knowledge, as this behaviour is implemented in methods of the class. The processing of knowledge is achieved by message passing between instances of a range of associated classes of objects.

So, based on the above example, at this moment in time, the MSE Moderator must have at least three Design Agent Module objects in its knowledge base, i.e. representing the Project Agent, the Manufacturing Resource Agent and the Layout Agent. Each Design Agent Module contains some static information about the MSE agent, for example:

1. the name of the MSE agent, (e.g. Project Agent)

2. how the MSE agent can be contacted, (this may be an email address, or an IP address, or details of some way in which a message can be passed to the agent) 
3. class names of objects the MSE agent might be interested in. (e.g. the Project Agent would be interested in any objects that are related to the objectives he has defined for the project: these may include estimates and predictions of product throughput from different processes or resources in the system design, and costs associated with different processes, resources and facilities.

The Design Agent Module is also linked to a knowledge base which provides detailed knowledge for the MSE Moderator to use when determining whether an MSE agent is interested in a particular change. The structuring of this detailed knowledge is complex as potentially it can take many different forms, depending on the expertise and functionality of the MSE agent, and the type of environment in which the project team are sharing information about the manufacturing system design. For this reason, the prototype implementation of the MSE Moderator has made use of the flexible KRM approach to structure this knowledge.

In the example, the MSE Agents are all sharing design information within the shared databases that are accessed via the Information Manager. The MSE Moderator therefore also collects information about design decisions that have been taken, via the Information Manager. Each Design Agent Module provides the MSE Moderator with a mental model of a particular Design Agent. Hence, the MSE Moderator may be associated with many different Design Agent Modules, and at any stage of the project, Design Agent Modules may be added, removed or modified. In addition to the static information about the MSE agent described above, the KRM has been used to provide the MSE Moderator with knowledge about each MSE Agent's area of interests. This is necessary so that it can decide whether any particular MSE Agent should be contacted and made aware of a current design decision. The type of knowledge the MSE Moderator requires can be demonstrated by considering the short MS Design Scenario given above. For example, the Design Agent Module for the Project Agent should let the MSE Moderator know that the Project Agent should be contacted if any throughput estimates or predictions are added to the database that are lower than specified in the project objectives. Similarly, knowledge in the Design Agent Module for the Project Agent Module should tell the MSE Moderator that the Project Agent needs to be made aware if predictions of production costs are higher than the costs specified in the objectives by the Project Agent. Hence, the knowledge in any Design Agent Module will depend on what is of importance to that particular MSE Agent. For example, the Manufacturing Resource Agent may want to be notified if any change is made to information related to any manufacturing resource he has specified for use in the new manufacturing system.

In this way, by using a Design Agent module to represent each MSE agent, the MSE Moderator is populated with knowledge about the knowledge used by the MSE agents that are currently participating in the project. The Design Agent Modules in the knowledge base therefore provide the MSE Moderator with the background knowledge that it requires to support the multi-discipline design team. As explained in section 2.2, the MSE Moderator performs two main functions, the Moderation Process and the Knowledge Acquisition Process.

\subsubsection{Moderation Process}

Most of the MSE Moderator's activities during the moderation process are performed in the background, and MSE Agents are probably not conscious of its presence until 
someone makes a design decision that directly affects them, and the MSE Moderator consequently contacts them. In order to test and demonstrate the prototype MSE Moderator, a trace screen was implemented, to display the activities that would normally be carried out in the background. This screen can be seen in figure 3 .

The moderation process is activated whenever a design decision is made, and this is identified by a change being made to the design information within the shared databases, i.e. in the prototype MISSION system whenever a change is made to the information held in the shared (Oracle) databases administered by the Information Manager, the moderation process begins. Design change identification is achieved by the MSE Moderator polling and asking the Information Manager for details of any changes that have taken place. The MSE Moderator program is also connected to a knowledge base (ObjectStore database), which contains the Design Agent Modules, and the MSE Moderator's Working Memory Object. The Moderator Working Memory class stores information which the MSE Moderator uses during the design moderation process. This includes details of the changed object, and other information to help manage its interactions with Design Agent Modules. Whenever a design change is identified, it must then be evaluated.

Consider again the given Manufacturing System Design Scenario. The Manufacturing Resource Agent has worked on the system design, and has specified production machinery which can satisfy the maximum capacity requirements that were defined in the project objectives. The machinery is to be installed in existing facilities, and the Layout Agent plans this part of the work, simulations are run and the required throughput can be achieved. The manufacturing system design is therefore progressing satisfactorily. However, later in the project, an unexpected problem arises over the installation of services, and the planned layout must be changed.

The MSE Moderator is routinely checking for design changes (working in the background as usual) and detects that a change has been made to information related to a manufacturing resource. It records details of the change in the Working Memory Object within its knowledge base (as usual). It then continues the moderation process, by checking the static information within each of the Design Agent Modules in turn, to see if any of the MSE Agents may be interested in the change. It identifies that the Manufacturing Resource Agent is potentially interested, so the MSE Moderator takes the further step of processing all the knowledge it has about the Manufacturing Resource Agent. During the course of processing this knowledge, it finds out that the Manufacturing Resource Agent is only interested in the change if the changed manufacturing resource was initially specified by this agent. The MSE Moderator therefore further interrogates the Information Manager to obtain more information about the resource, and compares the name of the specifier with the name of the MSE Agent, and finds they are the same. The MSE Moderator therefore contacts the Manufacturing Resource Agent, and notifies him of the design change, so that he can re-evaluate the design.

The Manufacturing Resource Agent may then revisit his simulation models, reassess the performance of the system, and find the predicted throughput is slightly reduced as a result of the changes. He should then modify this predicted value in the design database. Once again, the MSE Moderator would be routinely checking for design 
changes and would detect that a change has been made to a throughput prediction. Having recorded the change, and checked its knowledge in the current Design Agents' Modules, the Moderator could then identify that potentially the Project Agent may be interested in this change. Based on the detailed knowledge the MSE Moderator has in its Design Agent Module for the Project Agent, it would further interrogate the Information Manager and obtain more information about the required average and maximum throughputs of the system. If, for example, the modified throughput prediction was now less than the required minimum, the MSE Moderator would notify the Project Agent. Figure 3 shows a trace of these activities. At 15:05:32 the MSE Moderator was notified of the change, and checked the static information in its Design Agent Module for the Project Agent. It then identified that the Project Agent was potentially interested in changes of this type. The MSE Moderator therefore carried on with the next stage of its moderation process, which was to process the knowledge it holds about the Project Agent. It identified that the changes was likely to be significant for the Project Agent, and the MSE Moderator therefore contacted the Project Agent - as shown by the small window at the bottom of the figure.

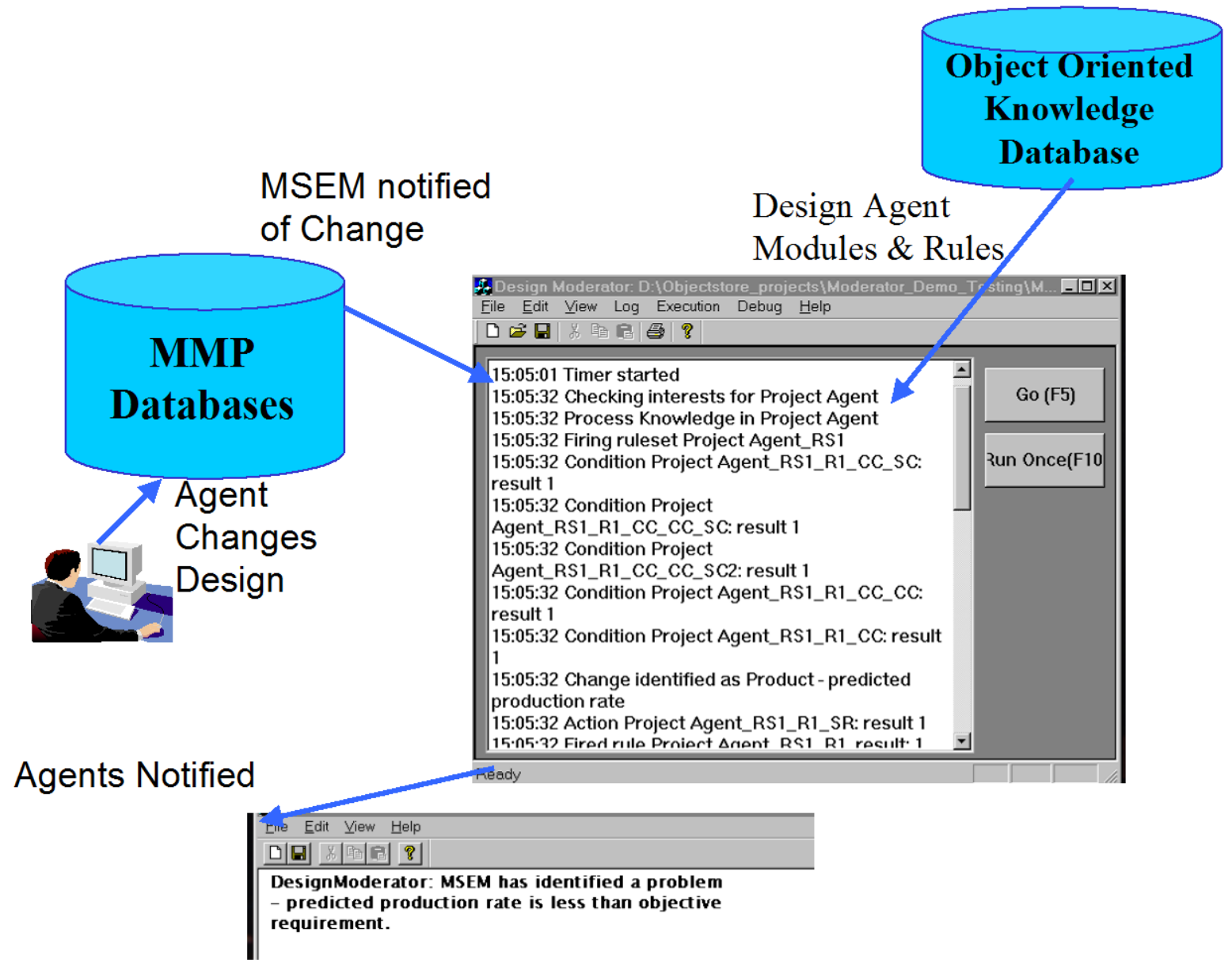

Figure 3: Design Moderation Module

The above description provides a small example of how the MSE Moderator may operate in a particular Manufacturing System Design scenario. In general, the activities in the moderation process can be summarised as follows. 
The MSE Moderator routinely checks for design changes (through the information manager). When a change is identified, it records it, and does a quick check of the static information stored for each Design Agent Module, to see if it is possible that the change may be significant to one or more of the MSE Agents. If it finds that the change is potentially significant, the MSE Moderator takes the further step of processing all the knowledge it has about the particular MSE Agent(s). When the MSE Moderator processes its knowledge of a particular MSE Agent, it may need to gather further information about other elements of the design, via the Information Manager. Using all these sources of potential information, the MSE Moderator will determine whether it is necessary to communicate with any of the MSE Agents. If it is necessary, details of how the MSE Agent can be contacted will be extracted from static information for the particular Design Agent Module, and an appropriate message sent to the MSE Agent.

The functionality defined in section 2.2 has therefore been achieved as follows:

1. The MSE Moderator must know whenever a change is made to the MS design:In the context of the MMP, the Information Manager signals the MSE Moderator whenever a change in the MSE design is recorded in the project database. The signal includes information to identify the elements of the design added or changed.

2. The MSE Moderator must be able to identify when a design change may cause conflict:- The MSE Moderator will therefore retain and apply knowledge about the knowledge used by each of the MSE agents. This is done using Design Agent Modules in its knowledge base. During the moderation process it is likely that the MSEM will request through the Information Manager information about design features related to the change. The MMP Data Model defines the structure used to identify related features within the MSE Design Database.

3. The MSE Moderator must communicate the detection of possible conflict to all MSE agents which it deems to have an interest in resolving the conflict, and when necessary remain in dialogue with these agents until resolution is achieved. Communication details are held in each Design Agent Module, and in the prototype implementations, communications via emails and via socket messages were used. Working memory facilitates continuing coherent dialogue as necessary.

\subsubsection{Knowledge Acquisition}

The MSE Moderator must be able to acquire knowledge which is generally applicable to the industry and enterprise, including the knowledge of all MSE agents currently participating in the project. In the prototype implementation of the MSE Moderator, the Knowledge Acquisition process has been implemented as an application, which may be used to create, delete or modify Design Agent Module objects, and their associated knowledge objects. The resulting objects are stored, as persistent objects in an object oriented knowledge database. Figure 4 shows the interface from the prototype MSE Moderator Knowledge Acquisition module. This can be used whenever new Agents join a project, or existing Agents are changed in any way.

An MSE Moderator functioning for a particular MSE project will acquire knowledge of the particular MSE agents available to and participating in the project, and be able to update that knowledge as the project progresses. 


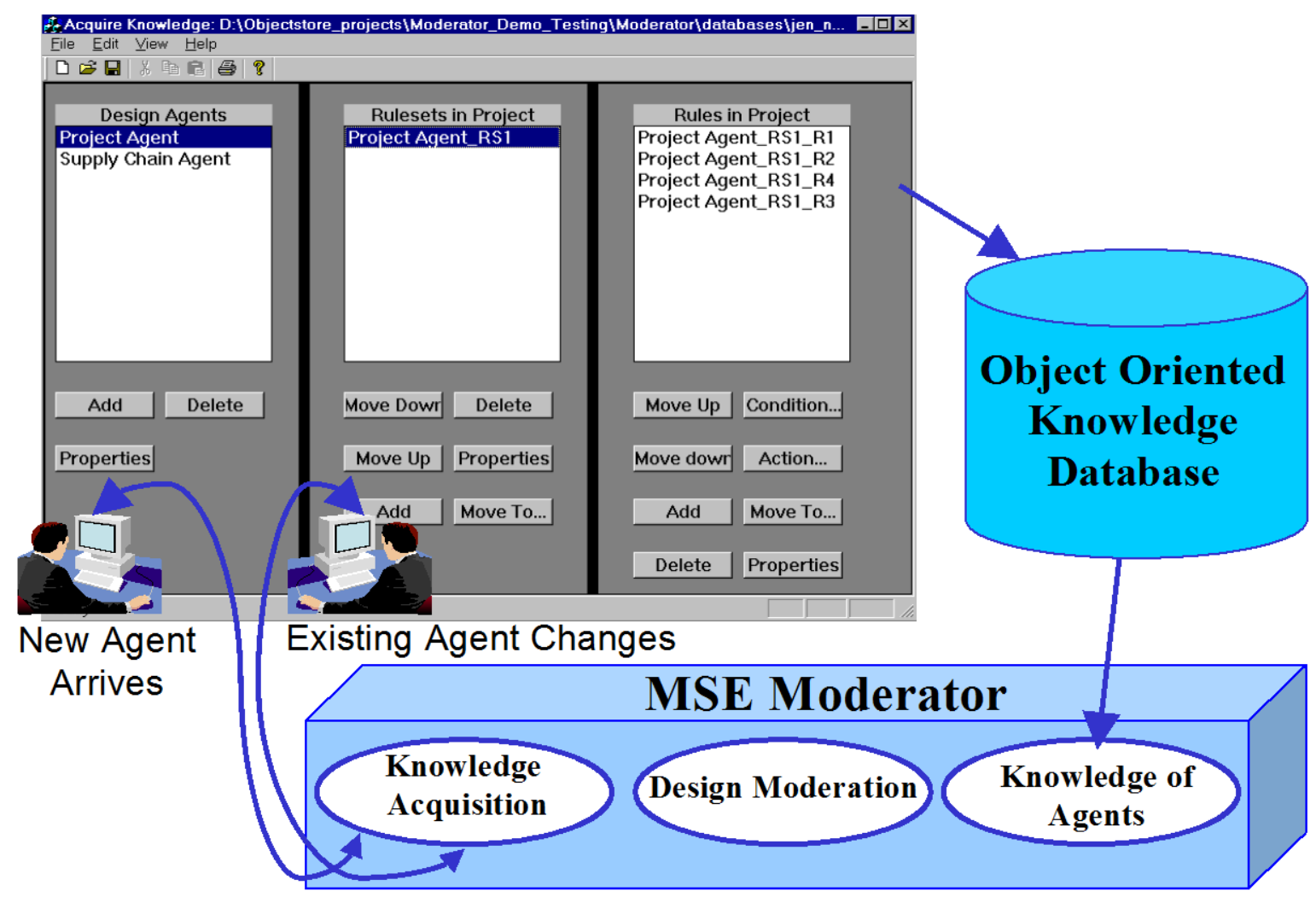

Figure 4: Knowledge Acquisition Module

\section{Conclusions}

A prototype MSE Moderator has been designed and implemented to demonstrate the feasibility of the MSE Moderator concepts within the MISSION MMP context. The prototype was successfully demonstrated to industrial collaborators in the final stages of the MISSION Research Project.

The MSE Moderator has been shown to work in the context of the MISSION MMP, but it has also been shown to have much wider applicability. There is now a need for research to establish a taxonomy of knowledge relevant to the process of MSE moderation, which in turn will lead to the definition of an effective methodology for capture and maintenance of moderation knowledge in a live implementation. There is also further scope for research into an MSE Moderator knowledge acquisition interface which is both user friendly, and which can learn from experiences gained in earlier MSE projects.

\section{References:}

Borja, V, Harding, J A, and Bell, R, (2001) 'A conceptual view on data model driven reverse engineering', International Journal of Production Research, 39(4), pp 667 687, ISSN 00207543.

Bouti A. and Ait Kadi, D., (1998), Capturing Manufacturing System Knowledge Using Multi-View Modelling, Int. J. Computer Integrated Manufacturing, Vol. 11, No. 1, pp. 77 -93. 
ESPRIT Project 688/5288, (1993), CIMOSA: Open System Architecture for CIM, AMICE, Volume 1, ESPRIT Consortium AMICE (Ed.), $2^{\text {nd }}$, revised and extended edition, Springer-Verlag.

Harding, J A, (1996), 'A Knowledge Representation Model to Support Concurrent Engineering Team Working', PhD Thesis, Loughborough University, UK.

Harding, J.A. and Popplewell, K., (1996), "Driving Concurrency in a Distributed Concurrent Engineering Project Team: A Specification for an Engineering Moderator", International Journal of Production Research, ISSN 0020 7543, 34(3), pp 841-861.

Harding, J A, Yu, B, and Popplewell, K, (1999), "Information Modelling: An Integration of Views of a Manufacturing Enterprise", International Journal of Production Research, ISSN 0020 7543, 37(12), pp 2777-2792.

Krause, F L, Kimura, F, Kjellberg, T, Lu, S C Y, (1993), "Product Modelling", Annals of the CIRP, Vol. 42(2) 695-706.

McKay, A., Bloor, M.S., de Pennington, A. (1996). A framework for product data, IEE Transactions on Knowledge and Data Engineering, Vol. 8, No. 5, October, pp. 825-838.

Mission Consortium (2001), "Final Report from MISSION Project - Deliverable D24”, K. Popplewell, K., Harding, J. A., Rabe, M. (Eds), MISSION website (www.ims-mission.de).

Molina, A., and Bell, R. (1999) A Manufacturing Model Representation of a Flexible Manufacturing Facility, Proceedings of the Institution of Mechanical Engineers, Vol. 213, Part B, pp. 225-246.

Omar, A R, Harding, J A, and Popplewell, K, (1999), "Design for Customer Satisfaction: An Information Modelling Approach", Integrated Manufacturing Systems, (The International Journal of Manufacturing Technology Management) ISSN 0957-6061, 10(4), pp 199-209.

Rabe, M. (2000), "Future of Simulation in Production and Logistics: Facts and Visions", in: Mertins, K.; Rabe, M. (Eds.): The New Simulation in Production and Logistics. 9th ASIM Dedictaed Conference on Simulation in Production and Logistics, Berlin 2000, pp. 21-43.

Scheer, A W, (1992), Architecture of Integrated Information Systems: Foundations of Enterprise Modelling, Springer-Verlag, Berlin.

Yu, B, Harding, J A, and Popplewell, K, (2000), 'A Reusable Enterprise Model', International Journal of Operations and Production Management , ISSN 0144-3577, 20(1), pp 50-69. 
A Manufacturing System Engineering Moderator: an aid for multi-discipline project teams

Revised for International Journal of Production Research

$1^{\text {st }}$ November 2002

Page 16 of 16 